\title{
SYNTHESIS OF SUPERVISORY CONTROLLERS FOR HYBRID SYSTEMS USING ABSTRACTION REFINEMENT
}

\author{
Olaf Stursberg \\ Process Control Laboratory (BCI-AST) \\ University of Dortmund, D-44221 Dortmund (Germany) \\ Email: olaf.stursberg@uni-dortmund.de
}

\begin{abstract}
For the computationally challenging task of synthesizing supervisory controllers for hybrid systems, this paper suggests to use guided abstraction refinement. Starting from a discrete abstraction of the hybrid model, so-called candidate paths are determined as possible controlled evolutions which satisfy given specifications for safety and goal attainment. A specialized validation procedure is used to check if the corresponding control strategies satisfy the specifications also for the original hybrid system. If not, the abstract model is refined according to the validation result. Since different validation methods (with different computational costs) are used, reachable hybrid sets have to be computed only for relatively few combinations of control inputs and locations of the hybrid automaton. Copyright (c) 2005 IFAC
\end{abstract}

Keywords: Abstraction, Automaton, Logic Control, Reachability, Synthesis.

\section{INTRODUCTION}

This paper addresses the task of computing supervisory control strategies for hybrid systems with nonlinear continuous dynamics. Supervisory control is here understood as the closed-loop setting, in which the hybrid plant generates an event when its continuous state reaches an event set, and the controller immediately returns a discrete control action which determines the further evolution of the hybrid plant. A number of approaches considering this task have been published recently, see e.g. (Asarin et al., 2000; Gonzalez et al., 2001; Koutsoukos et al., 2000; Koo et $a l ., 2001)$. Most of these approaches establish extensions of the supervisory control theory for discrete systems (Ramadge and Wonham, 1989) to hybrid models, and involve the step of determining which hybrid states are reachable under the influence of a discrete control action. Techniques to accomplish this step include level set methods (Trontis and Spathopoulos, 2003), viability methods (Bayen et al., 2002), and computing ellipsoidal or polyhedral inclusions of reach sets (Stursberg and Krogh, 2003). However, these techniques are known to be computationally expensive, and to scale badly with the dimension of the continuous state space - an objective for improving the efficiency of controller synthesis is thus to reduce reach set computation as much as possible.

For this reason, a synthesis approach employing the concept of guided abstraction refinement is proposed here. As described in the context of verification (Clarke et al., 2003), the principle is to use a discrete abstraction to search for evolutions (counterexamples) which violate a given property. Only for these evolutions the hybrid dynamics is investigated, in order to validate that the property is not satisfied for the hybrid model. As described in (Stursberg et al., 2003), applying a set of different validation methods with different computational costs can lead to the advantage that reach set computation is required only for a small portion of the state space. If the steps of computing counterexamples, validation, and re- 
fining the abstract model based on the validation result are applied iteratively, the analysis task can often be solved with a relatively low overall effort.

The concept is modified here for the purpose of controller synthesis: a discrete abstraction of the hybrid model is searched for candidate paths which encode control strategies that potentially fulfill safety and goal attainment specifications for the controlled system. The validation methods check if it is guaranteed that the hybrid evolutions, which correspond to the candidate path, meet the specifications. Also here, the outcome of the validation step is used to refine the abstract model (and thus affects the search for further candidate paths).

The method presented here is similar to the concept of l-complete abstractions, as described in (Moor et al., 2002). However, the type of abstraction as well as the methods for validation and refinement follow different principles.

\section{PROBLEM STATEMENT AND MODEL TRANSFORMATION}

The investigation considers the following type of hybrid automaton with discrete inputs:

Def. 1: A hybrid automaton with discrete inputs is given by $H A=(X, Z, V, \gamma, i n v, \Theta, g, r, f)$ with:

- the continuous state space $X \subseteq \mathbb{R}^{n_{x}}$ and $x \in X$;

- the finite set of locations $Z=\left\{z_{1}, \ldots, z_{n_{z}}\right\}$;

- the finite set $V=\left\{v_{1}, \ldots, v_{n_{d}}\right\}$ of discrete inputs $v_{j} \in \mathbb{R}^{n_{v}}$; an input availability mapping $\gamma: Z \rightarrow 2^{V} \backslash \emptyset$ assigns a set $V_{z} \subseteq V$ to $z \in Z$;

- the mapping inv $: Z \rightarrow 2^{X}$ which assigns a polyhedral invariant inv $\left(z_{j}\right)=\left\{x \mid \exists n_{p_{j}} \in\right.$ $\mathbb{N}, C_{j} \in \mathbb{R}^{n_{p_{j}} \times n_{x}}, d_{j} \in \mathbb{R}^{n_{p_{j}}}, x \in X: C_{j}$. $\left.x \leq d_{j}\right\}$ to each $z_{j} \in Z$;

- the finite transition set $\Theta \subseteq Z \times Z$; a transition from $z_{1} \in Z$ to $z_{2} \in Z$ is denoted by $\left(z_{1}, z_{2}\right)$;

- a mapping $g: \Theta \rightarrow 2^{X}$ that associates a guard $g\left(\left(z_{1}, z_{2}\right)\right) \subseteq X$ with each $\left(z_{1}, z_{2}\right) \in \Theta$ such that: $g\left(\left(z_{1}, z_{2}\right)_{j}\right)=\left\{x \mid \exists n_{g_{j}} \in \mathbb{N}, C_{j} \in\right.$ $\left.\mathbb{R}^{n_{g_{j}} \times n_{x}}, d_{j} \in \mathbb{R}^{n_{g_{j}}}: C_{j} \cdot x \leq d_{j}\right\}$. For all pairs of transitions $\left(z_{1}, \bullet\right) \in \Theta$, it is required that the corresponding guards are disjoint;

- a linear reset function $r: \Theta \times X \rightarrow X$, assigning $x^{\prime} \in X$ to each $\left(z_{1}, z_{2}\right) \in \Theta$ and $x \in g\left(\left(z_{1}, z_{2}\right)\right)$;

- a flow function $f: Z \times X \times V \rightarrow \mathbb{R}^{n}$ that defines a continuous vector field $\dot{x}=f(z, x, v)$ for each $z \in Z$.

The states $x_{k}:=x\left(t_{k}\right)$, locations $z_{k}:=z\left(t_{k}\right)$, and inputs $v_{k}=v\left(t_{k}\right)$ are defined on an ordered time set $T=\left\{t_{0}, t_{1}, t_{2}, \ldots\right\}, t_{k} \in \mathbb{R}^{\geq 0}$, which contains the initial time $t_{0}$ and all times at which transitions occur. For a given series of inputs $v_{k}$, a feasible run of $H A$ is a sequence $\phi_{\sigma}=$ $\left(\sigma\left(t_{0}\right), \sigma\left(t_{1}\right), \sigma\left(t_{2}\right), \ldots\right)$ of hybrid states $\sigma_{k}:=$ $\sigma\left(t_{k}\right)=\left(z_{k}, x_{k}\right)$ according to:

- $\sigma\left(t_{0}\right)=\left(z_{0}, x_{0}\right)$ with $z_{0}=z\left(t_{0}\right) \in Z$ and $x_{0} \in \operatorname{inv}\left(z_{0}\right), x_{0} \notin g\left(\left(z_{0}, \bullet\right)\right)$ for any $\left(z_{0}, \bullet\right) \in \Theta$.

- $\sigma\left(t_{k+1}\right)$ follows from $\sigma\left(t_{k}\right)$ by:

(i) continuous evolution: $\chi:[0, \tau] \rightarrow X$, $\tau \in \mathbb{R}^{>0}$, where $\chi\left(t_{0}\right)=x_{k}, \dot{\chi}(t)=$ $f\left(z_{k}, \chi(t), v_{k}\right)$ with an existing unique solution for $t \in[0, \tau]$, and $\chi(t) \in \operatorname{inv}\left(z_{k}\right)$, but for all $g\left(\left(z_{k}, \bullet\right)\right) \in \Theta$ and $t \in[0, \tau[$ : if $\chi(t) \in g\left(\left(z_{k}, \bullet\right)\right)$, no $t^{\prime}$ with $t<t^{\prime} \leq \tau$ exists such that $\chi\left(t^{\prime}\right) \notin g\left(\left(z_{k}, \bullet\right)\right)$;

(ii) discrete transition: $\left(z_{k}, z_{k+1}\right) \in \Theta, \chi(\tau) \in$ $g\left(z_{k}, z_{k+1}\right)$, and $x_{k+1}=r\left(\left(z_{k}, z_{k+1}\right), \chi(\tau)\right) \in$ $\operatorname{inv}\left(z_{k+1}\right)$

(iii) input selection: $v_{k+1} \in V_{z_{k+1}}$.

Two characteristics of this semantics are important: (a) A transition can occur for an arbitrary continuous state $\chi(\tau)$ in $g\left(\left(z_{k}, \bullet\right)\right)$, but it must be taken before the guard set is left. (This construction is suitable, e.g., to model by $g\left(\left(z_{k}, \bullet\right)\right)$ that the state which triggers the transition (a threshold) can only be measured with a limited precision.) (b) The discrete input $v_{k}$ can be changed with a transition, but is constant otherwise.

The control task for this setting is defined as follows:

Def. 2: Given are:

- an initial state set $\Sigma_{0}$ with $\left(z_{0}, x_{0}\right) \in \Sigma_{0}$, $z_{0} \in Z, x_{0} \in X_{0}$ with a bounded polyhedral set $X_{0} \subset \operatorname{inv}\left(z_{0}\right)$ that is disjoint with all $g\left(\left(z_{0}, \bullet\right)\right)$,

- a set $\Sigma_{F}=\left\{\Sigma_{F, 1}, \ldots, \Sigma_{F, n_{F}}\right\}$ of forbidden state sets $\Sigma_{F, j}$ with $(z, x) \in \Sigma_{F, j}$, such that $z \in Z, x \in X_{F, j}$ with a bounded polyhedron $X_{F, j} \subset i n v\left(z_{F, j}\right)$, and $\Sigma_{F, j} \cap \Sigma_{0}=\emptyset$,

- and a goal set $\Sigma_{G},\left(z_{G}, x\right) \in \Sigma_{G}$ with $z_{G} \in Z$, $x \in \operatorname{inv}\left(z_{G}\right)$, and $\Sigma_{G} \cup \Sigma_{F}=\emptyset$.

The synthesis task is to find a control strategy $\left(v_{0}, v_{1}, v_{2}, \ldots\right)$ for $H A$ such that the corresponding feasible run starting from every $\left(z_{0}, x_{0}\right) \in \Sigma_{0}$ leads into $\Sigma_{G}$ (goal attainment), while no state $(z, x) \in \Sigma_{F, j}, \Sigma_{F, j} \in \Sigma_{F}$ is encountered (safety property).

To simplify the further description, the open system $H A$ is rewritten as a closed system that includes all possible control strategies: Since any $v_{k} \in V_{z}$ can possibly be applied in $z \in Z$, the closed system is defined with an extended location set containing pairs $(z, v)$ with $v \in V_{z}$ :

Def. 3: For $H A$ as in Def. 1, the corresponding closed hybrid automaton is defined by: $H A^{c}=$ $\left(X^{c}, Z^{c}, i n v^{c}, \Theta^{c}, g^{c}, r^{c}, f^{c}\right)$ with:

- $X^{c}=X$;

- $Z^{c}=\left\{z_{1}^{c} ; \ldots, z_{n_{z}^{c}}^{c}\right\}$ where for each $z \in Z$ : $\forall v \in V_{z}: \exists z^{c}=(z, v) \in Z^{c}$; 
- $i n v^{c}: Z^{c} \rightarrow 2^{X}$ with $i n v^{c}\left(z^{c}\right)=i n v(z)$ for $z^{c}=(z, \bullet)$

- $\Theta^{c} \subseteq Z^{c} \times Z^{c}$ such that $\left(z_{1}^{c}, z_{2}^{c}\right) \in \Theta^{c}, z_{1}^{c}=$ $\left(z_{1}, \bullet\right), z_{2}^{c}=\left(z_{2}, \bullet\right)$ if $\exists\left(z_{1}, z_{2}\right) \in \Theta$

- $g^{c}: \Theta^{c} \rightarrow 2^{X}$ with $g^{c}\left(\left(z_{1}^{c}, z_{2}^{c}\right)\right)=g\left(\left(z_{1}, z_{2}\right)\right)$ for $z_{1}^{c}=\left(z_{1}, \bullet\right), z_{2}^{c}=\left(z_{2}, \bullet\right)$

- $r^{c}: \Theta^{c} \times X \rightarrow X$ with $r^{c}\left(\left(z_{1}^{c}, z_{2}^{c}\right), x\right)=$ $r\left(\left(z_{1}, z_{2}\right), x\right)$ for $z_{1}^{c}=\left(z_{1}, \bullet\right), z_{2}^{c}=\left(z_{2}, \bullet\right)$

- $f^{c}: Z^{c} \times X \rightarrow \mathbb{R}^{n}$ with $f^{c}\left(z^{c}, x\right)=f(z, x, v)$ for $z^{c}=(z, v), v \in V_{z}$.

The semantics for $H A^{c}$ with extended hybrid states $\left(z^{c}, x\right)$ is obvious from Def. 1 . The control task according to Def. 3 , is reformulated for $H A^{c}$ as follows: The initial set is changed to $\Sigma_{0}^{c}$ with $z_{0}^{c}=\left(z_{0}, v\right) \in \Sigma_{0}^{c}, v \in V_{z_{0}}, x_{0} \in X_{0} \subset$ $\operatorname{inv}\left(z_{0}\right)$. The goal set is modified to $\Sigma_{G}^{c}$ with $z_{G}^{c}=\left(z_{G}, v\right) \in \Sigma_{G}^{c}, v \in V_{z_{G}}, x \in \operatorname{inv}\left(z_{g}\right)$, and the forbidden set $\Sigma_{F}^{c}$ is altered correspondingly. The sets of locations which involve initial, goal, or forbidden states, are denoted by $Z_{0}^{c}, Z_{G}^{c}$, and $Z_{F}^{c}$ respectively. The control task renders then to transfer all states of $\Sigma_{0}^{c}$ with $z_{0}^{c}=\left(z_{0}, v\right)$ for one(!) $v \in V_{z_{0}}$ into $\Sigma_{G}^{c}$ while $\Sigma_{F}^{c}$ is never encountered.

The closed hybrid automaton is referred to as concrete model $C$, and to simplify notation, the index ${ }^{c}$ ' is omitted for $i n v^{c}, \Theta^{c}, g^{c}, r^{c}$, and $f^{c}$.

\section{SYNTHESIS BASED ON ABSTRACTION REFINEMENT}

The scheme of the procedure to synthesize a control strategy for $C$ is shown in Fig. 1, and can be summarized as follows: First, an abstract model $A^{(0)}$ is obtained from $C$ by an appropriate abstraction function (see Sec. 3.1). The latter essentially transfers the discrete dynamics of $C$ to $A^{(0)}$, abstracts from the continuous part, and accounts for the state sets which are relevant for the specification $\left(\Sigma_{0}^{c}, \Sigma_{G}^{c}, \Sigma_{F}^{c}\right)$. The next step searches for a candidate path $C P$ within the evolutions of $A^{(0)}$. A candidate path is a run of $A^{(0)}$ which leads from an abstract initial state to an abstract goal state, while not encountering an abstract forbidden state (Sec. 3.2). Since each abstract state in a candidate path corresponds to a location $z^{c}=(z, v)$ of $C$, it implicitly represents a control strategy $\left(v_{0}, v_{1}, v_{2}, \ldots\right)$. The following step of validation determines whether $C P$ can be realized for $C$ in the sense that every hybrid state reached along the corresponding abstract states in $C P$ is eventually transferred into the goal set. As further explained in Sec. 3.3, the validation comprises the application of three different methods with different computational costs in order to reduce the overall effort. If $C P$ is validated, a possible control strategy is found, and the procedure terminates (or can be resumed to search for another alternative strategy). If $C P$ is found to be invalid, the

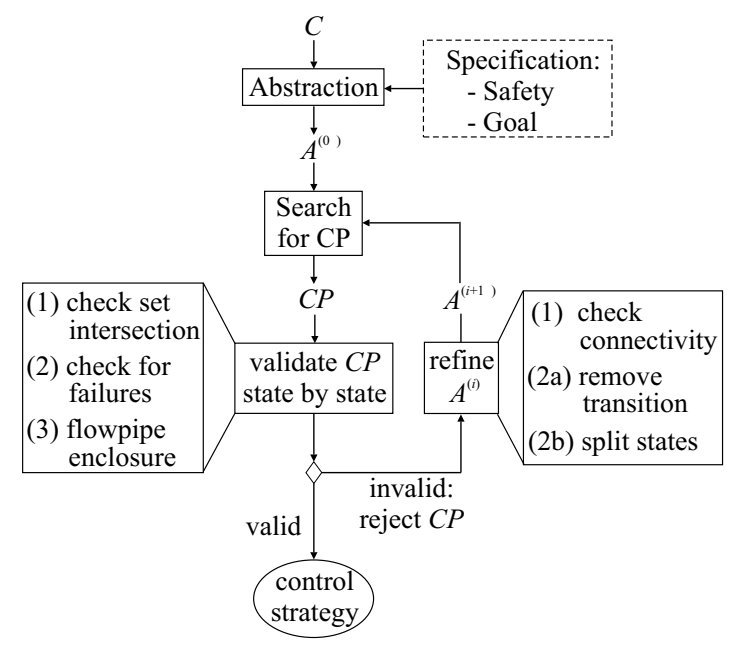

Fig. 1. The synthesis procedure.

strategy is rejected, and the procedure continues with a refinement step (Sec. 3.4). It first checks, if for the particular state of $C P$, in which the latter was found to be invalid, any evolution of $C$ which corresponds to the transition in $A^{(i)}$ exists at all (connectivity check). If not, the transition is removed from $A^{(i)}$ ( $i$ is the iteration index). An additional optional means to update $A^{(i)}$ is to split states according to the validation result, if the third validation method (flowpipe enclosure) was applied. It adds details to $A^{(i)}$ in the sense that reachability information obtained from evaluating $C$ is transferred to $A^{(i+1)}$.

The updated abstract model is searched for another candidate path, and the cycle of searching, validation, and refinement continues, until a suitable control strategy is found, no further candidate path can be determined, or an upper bound on the number of iterations is exceeded.

\subsection{Abstraction}

The abstraction step resembles the one used in (Clarke et al., 2003) in the context of verification, but with modifications to consider $\Sigma_{0}^{c}, \Sigma_{G}^{c}$, and $\Sigma_{F}^{c}$. First, a transition system is introduced according to $T S=\left(\hat{S}, \hat{S}_{0}, \hat{E}\right)$, consisting of a finite state set $\hat{S}$, an initial set $\hat{S}_{0} \subset \hat{S}$, and a finite transition set $\hat{E} \subseteq \hat{S} \times \hat{S}$. A run of $T S$ is given by $\hat{\phi}=\left(\hat{s}_{0}, \hat{s}_{1}, \hat{s}_{2}, \ldots\right)$ with $\hat{s}_{i} \in \hat{S}$ and $\left(\hat{s}_{i-1}, \hat{s}_{i}\right) \in \hat{E}$.

In order to obtain $A^{(0)}=\left(\hat{S}, \hat{S}_{0}, \hat{E}\right)$ as a model of the type $T S$ such it represents an abstraction of $C$, an abstraction function $\alpha: Z \times X \rightarrow \hat{S}$ is defined as follows:

(1) A state $\hat{s}_{0} \in \hat{S}_{0}$ is introduced for any $z^{c} \in Z_{0}^{c}$, and $\hat{s}_{0}$ represents all hybrid states $\left(z^{c}, x\right)$ of $C$ with $z^{c} \in Z_{0}^{c}$ and $x_{0} \in X_{0}$.

(2) $\hat{S}_{F}$ includes a state $\hat{s}_{F}$ for any $z^{c} \in Z_{F}^{c}$ such that it corresponds to all $\left(z^{c}, x\right)$ with $z^{c} \in Z_{F, j}^{c}$ and $x \in X_{F, j}$ 
(3) $\hat{S}$ follows from $\hat{S}:=\hat{S}^{\prime} \cup \hat{S}_{0} \cup \hat{S}_{F}$ with $\hat{S}^{\prime}$ such that $\exists \hat{s} \in \hat{S}^{\prime}$ for any $z^{c} \in Z^{c} ; \hat{s} \in \hat{S}^{\prime}$ represents the states $\left(z^{c}, x\right)$ with: $x \in \operatorname{inv}\left(z^{c}\right)$ if $z^{c} \notin Z_{F}^{c}$, or $x \in \operatorname{inv}\left(z^{c}\right) \backslash X_{F, j}$ if $z^{c} \in Z_{F}^{c}$.

The set of abstract goal states $\hat{S}_{G}$ contains the states $\hat{s} \in \hat{S}$ which are assigned to $z_{G}^{c} \in Z_{G}^{c}$. The set $\hat{E}$ of $A$ contains transitions $\left(\hat{s}_{i}, \hat{s}_{l}\right)$ for any of the following cases with $\hat{s}_{i}=\alpha\left(z_{i}^{c}, x_{i}\right)$, $\hat{s}_{l}=\alpha\left(z_{l}^{c}, x_{l}\right)$ :

- $\exists\left(z_{i}^{c}, z_{l}^{c}\right) \in \Theta^{c}$,

- $\hat{s}_{i} \in \hat{S}_{0}$ and $z_{i}^{c}=z_{l}^{c} \in Z_{0}^{c}$,

- $\hat{s}_{l} \in \hat{S}_{F}, z_{i}^{c}=z_{l}^{c} \in Z_{F}^{c}$, and $x_{i} \in \operatorname{inv}\left(z_{F, j}\right) \backslash$ $X_{F, j}, x_{l} \in X_{F, j}$.

These assignments map every hybrid state of $C$ onto at least one discrete state of $A$, and each transition of $C$ has a correspondence in $A$. Thus, also every run of $C$ corresponds to a run of $A$, such that the latter is an abstraction of the hybrid model.

\subsection{Search for a Candidate Path}

A candidate path can now be defined as a particular run of $A$ :

Def. 4: Given the model $A$ with the sets $\hat{S}_{0}$, $\hat{S}_{F}$, and $\hat{S}_{G}$, a candidate path of $A$ is a run $C P=\left(\hat{s}_{0}, \hat{s}_{1}, \ldots, \hat{s}_{p}\right)$ with $\hat{s}_{0} \in \hat{S}_{0}, \hat{s}_{p} \in \hat{S}_{G}$, and $\hat{s}_{j} \notin \hat{S}_{F}$ for all $j \in\{0,1, \ldots, p\}$.

To determine $C P$, a standard reachability algorithm using breadth-first search is applied. It returns one of the shortest candidate paths existing for the current abstract model in each iteration.

\subsection{Validation}

The validation aims to check for every pair $\left(\hat{s}_{k}, \hat{s}_{k+1}\right)$ of subsequent states of $C P$ (and the intermittent transition $\left(\hat{s}_{k}, \hat{s}_{k+1}\right) \in \hat{E}$ ) if a corresponding behavior of $C$ exists in the following sense: In Fig. 2, let the set $I \subseteq i n v\left(z_{k}^{c}\right)$ mark the set of hybrid states represented by $\hat{s}_{k}$, and $\operatorname{inv}\left(z_{k+1}^{c}\right)$ the set represented by $\hat{s}_{k+1}$. In order to validate the pair $\left(\hat{s}_{k}, \hat{s}_{k+1}\right)$ of $C P$ as being a feasible step of a control strategy, each continuous state $x \in I$ must be transformed into $\operatorname{inv}\left(z_{k+1}^{c}\right)$. This requires that the continuous dynamics $f\left(z_{k}^{c}, x\right)$ must first transfer $x \in I$ into a state $x^{\prime}$ in the guard set $g\left(\left(z_{k}^{c}, z_{k+1}^{c}\right)\right)$, and applying the reset $x^{\prime \prime}=r\left(\left(z_{k}^{c}, z_{k+1}^{c}, x^{\prime}\right)\right)$ must yield a state $x^{\prime \prime} \in \operatorname{inv}\left(z_{k+1}^{c}\right)$. This refers to the case marked by (b) in Fig. 2; if the case (a) occurs, i.e. the trajectory does, e.g., not enter the guard set, then the step $\left(\hat{s}_{k}, \hat{s}_{k+1}\right)$ of $C P$ does not encode a feasible control strategy.

The validation scheme follows the objective to determine with an as small as possible effort whether
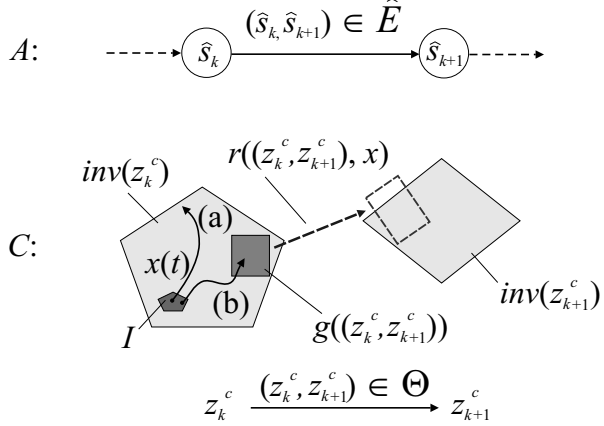

Fig. 2. Validating a transition of $A$ for $C$.

a candidate path does not encode a feasible control strategy. Thus, a series of validation methods with an increasing effort to reject a candidate path is applied, but the methods are increasingly successful in invalidating a transition of $C P$.

The first method, referred to as set intersection in Fig. 1, checks the necessary condition that $r\left(\left(z_{k}^{c}, z_{k+1}^{c}, x\right)\right)$ maps at least one $x \in g\left(\left(z_{k}^{c}, z_{k+1}^{c}\right)\right)$ into $\operatorname{inv}\left(z_{k+1}^{c}\right)$. For a linear reset function and a polyhedral guard set (see Def. 1), this check can be carried out relatively easy by a linear transformation of $g\left(\left(z_{k}^{c}, z_{k+1}^{c}\right)\right)$ and by intersecting the result with the polyhedron $i n v\left(z_{k+1}^{c}\right)$.

The second validation method (listed as failure check in Fig. 1), represents the key step in efficiently rejecting an invalid candidate path. It poses an optimization problem that searches for a trajectory which starts in $I$, but does not eventually lead to $\operatorname{inv}\left(z_{k+1}^{c}\right)$ through the transition $\left(z_{k}^{c}, z_{k+1}^{c}\right) \in \Theta$. Let $T=\left\{x^{\prime} \mid x^{\prime \prime} \in \operatorname{inv}\left(z_{k+1}^{c}\right)\right.$ : $\left.r^{-1}\left(x^{\prime \prime}\right)=\left(\left(z_{k}^{c}, z_{k+1}^{c}\right), x^{\prime}\right), x^{\prime} \in g\left(\left(z_{k}^{c}, z_{k+1}^{c}\right)\right)\right\}$ denote a target set, as the subset of the guard which is mapped into $\operatorname{inv}\left(z_{k+1}^{c}\right)$ by $r$. For each continuous trajectory starting in $x_{0} \in I$, the terminal state is defined as $x\left(t_{f}\right)=x_{0}+\int_{t_{0}}^{t_{f}} f\left(z_{k}^{c}, x(\tau)\right) d \tau$ such that one of the following criteria applies:

(1) $x\left(t_{f}\right) \in T$,

(2) $x\left(t_{f}\right) \in g\left(\left(z_{k}^{c}, z_{q}^{c}\right)\right)$ with $z_{q}^{c} \notin z_{k+1}^{c}$,

(3) $x\left(t_{f}\right) \in X_{F, j}$ for any $F_{j}$,

(4) $x\left(t_{f}\right) \notin \operatorname{inv}\left(z_{k}^{c}\right)$, but $\lim _{t \rightarrow t_{f}} x(\tau) \in \operatorname{inv}\left(z_{k}^{c}\right)$

(5) $x\left(t_{f}\right) \in \operatorname{inv}\left(z_{k}^{c}\right)$ and $t \geq t_{\max }$.

Additionally it is required that for $\tau \in\left[0, t_{f}[\right.$ none of the sets in (1) to (3) is hit by $x(\tau)$. The parameter $t_{\max } \in \mathbb{R}^{>0}$ denotes an upper time bound. Essentially, (1) refers to the desired case that $\operatorname{inv}\left(z_{k+1}^{c}\right)$ is reached, while the four other case render $\left(\hat{s}_{k}, \hat{s}_{k+1}\right)$ invalid, since either a different guard set, a forbidden region, or the border of the invariant is reached, or $t_{\max }$ is elapsed. To determine if any of the cases (2) to (5) applies for any $x_{0} \in I$, the following optimization problem is posed:

$$
\max _{x_{0} \in I}\left\|x\left(t_{f}\right)-x_{\text {cent }}\left(g\left(\left(z_{k}^{c}, z_{k+1}^{c}\right)\right)\right)\right\|_{2},
$$


with $x_{\text {cent }}\left(g\left(\left(z_{k}^{c}, z_{k+1}^{c}\right)\right)\right)$ denoting the center point of the guard set. The optimization is solved by nonlinear programming, in which the simulation of the continuous dynamics of $C$ is embedded, and the simulation terminates according to the criteria for $x\left(t_{f}\right)$ as given above.

If the optimization according to Eq. 1 leads to the result that $C P$ has to be rejected, the synthesis procedure continues with refinement (Sec. 3.4). If the validation was successful, the method flowpipe enclosure is applied to the validated step of $C P$. This method for computing reachable sets, which is in detail described in (Stursberg and Krogh, 2003), generates a sequence of hyper-rectangular polyhedra which completely enclose all continuous states in $i n v\left(z_{k}^{c}\right)$ that can be reached from $I$ by continuous evolution. The reason for applying this method is that the entry set for the location $z_{k+1}^{c}$ can be obtained from intersecting the enclosing polyhedra with $g\left(\left(z_{k}^{c}, z_{k+1}^{c}\right)\right.$, and applying the reset function $r\left(\left(z_{k}^{c}, z_{k+1}^{c}, x^{\prime}\right)\right)$ afterwards. Thus, this step produces the starting point for validating the next transition of $C P$.

\subsection{Refinement}

Three different situations have to be considered for refining $A^{(i)}$ in order to obtain an updated abstract model $A^{(i+1)}$ :

(1.) If the method of checking for set intersection revealed that, for a transition $\left(\hat{s}_{k}, \hat{s}_{k+1}\right)$ of $C P$, the corresponding transition $\left(z_{k}^{c}, z_{k+1}^{c}\right) \in \Theta$ of $C$ can never be taken, $\left(\hat{s}_{k}, \hat{s}_{k+1}\right)$ is removed from the set $\hat{E}$ of $A^{(i)}$.

(2.) If the method failure check returned the result that the transition $\left(\hat{s}_{k}, \hat{s}_{k+1}\right)$ of $A^{(i)}$ is invalid, the transition cannot not be removed immediately from $\hat{E}$, since a smaller entry set into $\operatorname{inv}\left(z_{k}^{c}\right)$ (through a different path) may lead to a positive validation result. However, if no continuous trajectory corresponding to $\left(\hat{s}_{k}, \hat{s}_{k+1}\right)$ can be found, this transition is eliminated from $\hat{E}$. The investigation of the non-existence of such a corresponding trajectory (named connectivity check in Fig. 1) can be numerically performed by the optimization described in (Stursberg et al., 2003). If a connecting trajectory is found but $\left(\hat{s}_{k}, \hat{s}_{k+1}\right)$ of $A^{(i)}$ is invalid, $C P$ is marked as rejected to exclude it from the further investigation.

(3.) If $\left(\hat{s}_{k}, \hat{s}_{k+1}\right)$ has been validated, and the method flowpipe enclosure has returned an entry set $I$ for the location $z_{k+1}^{c}$, refinement of $A^{(i)}$ by state splitting can be performed optionally. This means that the state $\hat{s}_{k+1}$ is replaced in $\hat{E}$ by two states $\hat{s}_{a}$ and $\hat{s}_{b}$, where $\hat{s}_{a}$ represents all hybrid states with $z_{k+1}^{c}$ and $x \in I$, and $\hat{s}_{b}$ corresponds to all hybrid states with $z_{k+1}^{c}$ and $x \in \operatorname{inv}\left(z_{k+1}^{c}\right) \backslash I . \hat{s}_{a}$ has the same ingoing and outgoing transitions as $\hat{s}_{k+1}$. Also for $\hat{s}_{b}$, the set of outgoing transitions is identical to the one of $\hat{s}_{k+1}$, but the set of ingoing transitions is reduced by the transition from $\hat{s}_{k}$, corresponding to the result of the flowpipe computation. (The refinement function $\alpha$ is modified accordingly.)

\section{APPLICATION TO A REACTOR SYSTEM}

To illustrate the approach, it is applied to the startup procedure of a continuous chemical liquidphase reactor. The system comprises a stirred tank with two inlets, one outlet, a cooling jacket, and a heating device. An exothermic reaction of two components $D_{1}$ and $D_{2}$ form a product $D_{3}$, and $D_{2}$ is supplied in excess. The task is to find a control strategy that drives the reactor from a state of being almost empty and at low temperature to nominal operation (filled, high temperature, and high yield). The relevant state variables are the liquid level $\left(x_{1}\right)$, the temperature of the mixture $\left(x_{2}\right)$, and the concentration $\left(x_{3}\right)$ of component $D_{1}$. A vector $v:=\left(F_{2}, F_{3}, K, H\right)^{\mathrm{T}}$ denotes the four discrete inputs: the valve settings for the inlet of component $D_{2}\left(F_{2}\right)$ and for the outlet $\left(F_{3}\right)$, as well as the status of the cooling $(K)$ and heating device $(H)$. Each input can be switched between two values (low/high or on/off) such that 16 different input combinations are available. The hybrid dynamics is modelled by:

$$
\begin{gathered}
\dot{x}_{1}=k_{1}+F_{2}-F_{3}, \\
\dot{x}_{2}=\left(k_{2}\left(k_{3}-x_{2}\right)+F_{2}\left(k_{4}-x_{2}\right)\right) / x_{1} \\
+k_{5}\left(k_{6}-x_{2}\right)\left(k_{7} / x_{1}+k_{8}\right) K \\
\dot{x}_{3}=\left(k_{9}-\left(k_{10}+F_{2}\right) x_{3}\right) / x_{1}
\end{gathered}
$$

but for the locations with $x_{1} \geq 0.8$ an additional term for $\dot{x}_{2}$ has to be considered:

$$
\dot{x}_{2}^{\prime}=\dot{x}_{2}+k_{11}\left(k_{12}-x_{2}\right)\left(k_{13}-k_{14} / x_{1}\right) H
$$

and for locations with $\left(0, k_{15}, k_{16}\right) \cdot x \geq k_{17}$ the following reaction terms are relevant:

$$
\begin{gathered}
\dot{x}_{2}^{\prime \prime}=\dot{x}_{2}^{\prime}+x_{3}\left(k_{18}+k_{19} x_{2}^{2}\right) \\
\dot{x}_{3}^{\prime}=\dot{x}_{3}+k_{20} \cdot \exp \left(k_{21} / x_{2}\right)
\end{gathered}
$$

(with constant parameters $k_{i}$ ). The 3 -dim. continuous state space is additionally partitioned by a second threshold for $x_{1}$, and two more thresholds for $x_{2}$. The invariant and guard sets of the hybrid automaton follow from this partitioning. The initial hybrid model $H A$ comprises 12 locations, 22 transitions, and seven forbidden sets (representing too high temperatures and liquid levels). The available input sets $V_{z}$ for each location $z$ are restricted to those inputs that are physically reasonable. Thus, the set $Z^{c}$ of locations of $H A^{c}$ contains 32 locations. 
When applying the synthesis procedure proposed in this paper, a feasible control strategy is found by the $17^{\text {th }}$ candidate path. The overapproximated continuous reachable state set that for this strategy is shown in Fig. 3, and obviously all trajectories emerging from the initial set eventually lead to the goal set. The figure indicates six different phases $\left(p_{1}\right.$ to $\left.p_{6}\right)$, each of which refers to a period of time with constant discrete input. The input for this strategy are: $v_{1}=v_{2}=(1,0,0,0)^{\mathrm{T}}$ (i.e., high value for $F_{2}, F_{3}$ closed, cooling and heating switched off), $v_{3}=v_{4}=(0,1,0,1)^{\mathrm{T}}$ (low value for $F_{2}, F_{3}$ open, cooling off, heating on), $v_{5}=(0,1,0,0)^{\mathrm{T}}$, and $v_{6}=(0,1,1,0)^{\mathrm{T}}$. The first 16 candidate path were invalidated by the failure check method. The synthesis result was obtained in approx. 4 minutes on a PC with P4 processor $(1.5 \mathrm{GHz})$. It has to be mentioned that the computation time and the number of candidate paths rejected before finding a feasible solution does largely depend on the ordering of the sets $V_{z}$. (If, e.g., this order is rearranged such that above control strategy corresponds to the first investigated candidate path, the algorithm terminates already after 40 seconds.)

\section{CONCLUSION}

The proposed approach has the following advantages in comparison to methods which explore the hybrid state space in a breadth-first style by applying each available input in each location (starting from $z_{0}$ ): (a) The search is restricted to the paths for which the discrete dynamics signals success, and the search is performed in a depthfirst style. (b) The validation method failure check was found to be very efficient in rejecting invalid strategies. (c) The refinement of $A$ by eliminating transitions can exclude several further invalid candidate paths. An open point at this stage, and thus matter of current investigation, is whether

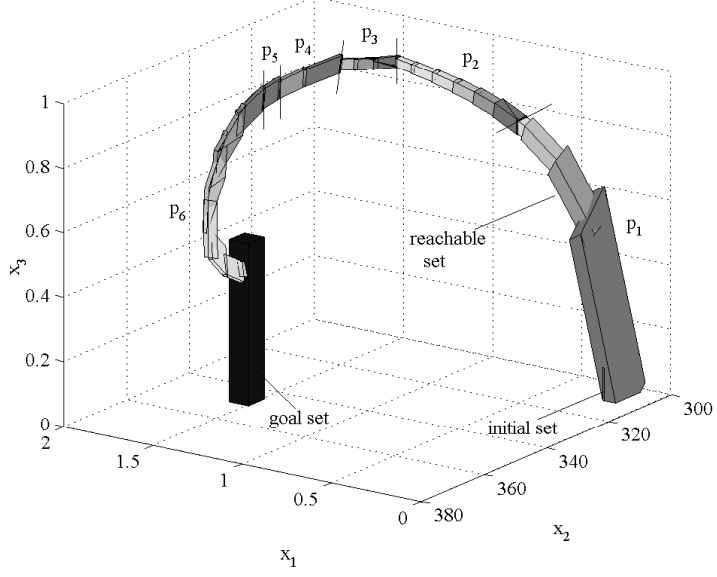

Fig. 3. Reachable continuous state set for the synthesized control strategy. the refinement by state splitting is indeed beneficial. It is also investigated if efficient heuristics can be developed for the order of choosing the discrete inputs (during the search for $C P$ ), depending on the outcome of preceding validations.

\section{REFERENCES}

Asarin, E., O. Bournez, T. Dang, O. Maler and A. Pnueli (2000). Effective synthesis of switching controllers for linear systems. Proc. of the IEEE 88(7), 1011-1025.

Bayen, A.M., E. Crueck and C. Tomlin (2002). Guaranteed overapproximations of unsafe sets for continuous and hybrid systems. In: Hybrid Systems - Comp. and Control. Vol. 2289 of LNCS. Springer. pp. 90-104.

Clarke, E.M., A. Fehnker, Z. Han, B.H. Krogh, O. Stursberg and M. Theobald (2003). Verification of hybrid systems based on counterexample-guided abstraction refinement. In: Tools and Algor. for the Constr. and Analysis of Systems. Vol. 2619 of LNCS. Springer. pp. 192-207.

Gonzalez, J.M.E., A.E.C Cunha J.E.R. Cury and B.H. Krogh (2001). Supervision of eventdriven hybrid systems: Modeling and synthesis. In: Hybrid Systems - Comp. and Control. Vol. 2034 of LNCS. Springer. pp. 247-260.

Koo, T.-J., G.J. Pappas and S. Sastry (2001). Mode switching synthesis for reachability specifications. In: Hybrid Systems - Comp. and Control. Vol. 2034 of LNCS. Springer. pp. 333-346.

Koutsoukos, X., P.J. Antsaklis, J.A. Stiver and M.D. Lemmon (2000). Supervisory control of hybrid systems. Proc. of the IEEE 88(7), 1026-1049.

Moor, T., J.M. Davoren and J. Raisch (2002). Strategic refinements in abstraction based supervisory control of hybrid systems. In: Proc. 6th Int. Workshop on Discrete Event Systems. pp. 329-334.

Ramadge, P.J.G. and W.M. Wonham (1989). The control of discrete event systems. Proc. of the IEEE 77(1), 81-98.

Stursberg, O., A. Fehnker, Z. Han and B.H. Krogh (2003). Specification-guided analysis of hybrid systems using a hierarchy of validation methods. In: Proc. 15th IFAC Conf. Analysis and Design of Hybrid Sys.. pp. 289-295.

Stursberg, O. and B.H. Krogh (2003). Efficient representation and computation of reachable sets for hybrid systems. In: Hybrid Systems - Comp. and Control. Vol. 2623 of LNCS. Springer. pp. 482-497.

Trontis, A. and M.P. Spathopoulos (2003). Hybrid control synthesis for eventuality specifications using level set methods. Int. J. of Control 76(16), 1599-1627. 